\title{
Influencia del docente y la docente, la metodología y el contenido de las clases de educación física en la percepción de estudiantado chileno
}

\author{
Influence of Teachers, Methodology and Content of Physical Education Classes in the \\ Perception of Chilean Students
}

\section{Influência do docente, metodologia e conteúdo das aulas de Educação Física na percepção dos alunos chilenos}

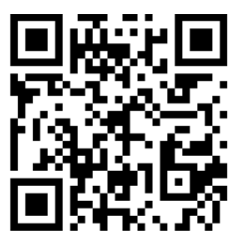

Frano Giakoni-Ramírez Universidad Autónoma de Chile

Temuco, Chile

Frano.giakoni@uautonoma.cl (iD) https://orcid.org/0000-0002-2685-8991

Daniel Duclos-Bastías Pontificia Universidad Católica de Valparaíso Valparaíso, Chile daniel.duclos@pucv.cl (D) https://orcid.org/0000-0002-9020-5776

David Parra-Camacho Universidad de Valencia Valencia, España david.parra-camacho@uv.es https://orcid.org/0000-0001-6002-1354

Recibido • Received • Recebido: 05 / 11 / 2019

Corregido • Revised • Revisado: 27 / 01 / 2021

Aceptado • Accepted • Aprovado: 28 / 02 / 2021

\begin{abstract}
Resumen:
Introducción. La relevancia de la educación en la sociedad actual es evidente, por ello, es de vital importancia conocer la percepción de los diversos actores que interactúan en esta, en particular, la del estudiantado sobre las metodologías y contenidos que ofrece el profesorado es determinante en el proceso de enseñanza-aprendizaje. Objetivo. Conocer la percepción que reportan estudiantes de tres centros educativos de la región de Valparaíso (Chile), en relación con la satisfacción por las actividades deportivas, la clase de educación física y la función del personal docente. Metodología. Se utilizó una escala de 12 ítems agrupadas en tres dimensiones. Se aplicó una escala de percepción a una muestra compuesta de 660 estudiantes de $7 \circ$ (NB5) y $8^{\circ}$ (NB6) de Enseñanza General Básica, pertenecientes a tres centros educativos chilenos (municipal, subvencionado y particular). Resultados. Se comprueba que las variables sexo y centro de escolarización influyen significativamente en la percepción del
\end{abstract}


http://doi.org/10.15359/ree.25-2.10

http://www.una.ac.cr/educare

educare@una.ac.cr

estudiantado sobre la asignatura. Conclusión. Estos resultados buscan mejorar las actuaciones de los diferentes agentes participantes en el proceso educativo, destinados a la mejora de la percepción del estudiantado con el fin de reforzar hábitos deportivos y de vida saludable.

Palabras claves: Educación Física; actitud del estudiante; actitud docente; ambiente de la clase.

\begin{abstract}
Introduction. The relevance of education in today's society is evident; therefore, it is of vital importance to know the perception of the various actors that interact in it, in particular, the perception of students about the methodologies and contents offered by the teacher is a determining factor in the teaching-learning process. Objective. To know the students' perception from three educational centers of the Valparaíso Region (Chile) in relation to the satisfaction with sports activities, the Physical Education class, and the teacher's role. Methodology. A scale of 12 items grouped in three dimensions was used. A perception scale was applied to a sample of 660 students of 7th (NB5) and 8th (NB6) grades of General Basic Education, belonging to three Chilean educational establishments (municipal, subsidized, and private schools). Results. It is proved that the variables sex and schooling center significantly influence the students' perception of the subject. Conclusion. These results seek to improve the actions of the different agents involved in the educational process, aimed at improving students' perception in order to reinforce sports and healthy lifestyle habits.
\end{abstract}

Keywords: Physical Education; student attitude; teacher attitude; classroom environment.

\begin{abstract}
Resumo
Introdução. A relevância da educação na sociedade atual é evidente, portanto, é de vital importância conhecer a percepção dos vários atores que nela interagem, em particular, a percepção de estudantes sobre as metodologias e conteúdos oferecidos pelo corpo docente é crucial no processo de ensino-aprendizagem. Objetivo. Conhecer a percepção relatada por estudantes de três centros educativos da Região de Valparaíso (Chile), em relação à satisfação das atividades desportivas, a aula de Educação Física e do papel do professor. Metodologia. Foi utilizada uma escala de 12 itens agrupados em três dimensões. Também foi aplicada uma escala de percepção a uma amostra de 660 estudantes do $7 \circ$ (NB5) e $8^{\circ}$ (NB6) ano do Ensino Básico Geral, pertencentes a três instituições de ensino chilenos (municipais, subsidiados e privados). Resultados. Está provado que as variáveis sexo e centro escolar têm uma influência significativa na percepção dos estudantes sobre a matéria. Conclusão. Estes resultados procuram contribuir com as ações dos diferentes agentes envolvidos no processo educativo, objetivando melhorar a percepção dos estudantes, a fim de reforçar os hábitos desportivos e de vida saudável.
\end{abstract}

Palavras-chave: Educação física; atitude do estudante; atitude do corpo docente; ambiente de sala de aula.

\title{
Introducción
}

En Chile, la Ley General de Educación (Ministerio de Educación, 2009) indica que educación es el proceso que constituye un continuo aprendizaje, el cual se mantiene durante toda la vida y que tiene como objetivo el desarrollo integral de las personas. Por ello, la educación física 
http://doi.org/10.15359/ree.25-2.10

se presenta como el ámbito idóneo para la adquisición de estilos de vida activos y saludables, favorecedor del desarrollo físico y motor (Rosa Guillamón et al., 2018).

En lo conceptual, la educación física cuenta con gran cantidad de acepciones, influenciadas por las modificaciones terminológicas de los términos ser humano, cuerpo, educación y sociedad (González Correa y González Correa, 2010). Parlebas (2001) la define como "una práctica de intervención que ejerce sobre las conductas motrices una influencia en función de normas educativas implícitas o explícitas" (p. 172).

Desde este punto de vista, el individuo descubre el mundo mediante la acción de su cuerpo, dando respuesta a sus propias necesidades mediante la relación con el entorno (González Correa y González Correa, 2010). De esta forma se obtiene información del mundo, y se elaboran internamente estructuras cognitivas y motrices necesarias para sustentar los futuros aprendizajes (Mendiara Rivas, 2008).

Por tanto, la educación física pretende un desarrollo armónico de la persona, desarrolla o restablece, mediante un abordaje corporal, las capacidades del individuo (Sassano y Bottini, 2000).

Se pueden determinar algunos de los aspectos que influyen en la educación física, de índole muy diversa y que le aportan un gran valor, tal y como señalan Sánchez-Oliva et al. (2015), la educación física influye positivamente en la percepción del estudiantado respecto a la actividad física en general, no únicamente a la realizada en los centros educativos, al fomentar, como consecuencia, estilos de vida activos y saludables. El incentivo hacia el deporte y la actividad física debe ser la principal finalidad de la educación física en el contexto escolar, tanto en niños, niñas como en adolescentes (Piéron et al., 2008). Destacan la interrelación del estudiantado como una de las características más relevantes de la educación física, al permitir la confección de determinadas actitudes y comportamientos que favorecen un ambiente óptimo para el desarrollo del estudiantado.

Por otro lado, Moreno et al. (2011) afirman que la importancia otorgada por el estudiantado a la clase de educación física está influenciada por factores muy diversos, entre los que destaca la motivación. Esta permite un disfrute y desarrollo de actitudes positivas hacia la práctica cuando es autodeterminada; sin embargo, la clase puede llegar a reportar actitudes negativas, tales como el aburrimiento, cuando no está presente tal motivación (Sánchez-Oliva et al., 2015).

Otros factores que afectan en la motivación son las creencias, valores, influencias sociales, apoyo familiar, así como los hábitos físicos y deportivos del entorno más próximo, pudiendo generar actitudes positivas, o negativas, hacia la práctica (Kane, 2015; Méndez-Giménez et al., 2013). En algunos estudios, como los realizados por González y Otero (2005), queda en evidencia que niños, niñas y adolescentes escolares que cuentan con una familia que realiza actividad física constante estarán con un mayor entusiasmo e interés por la práctica deportiva, y lograrán un cambio en la percepción y valoración de la educación física en el contexto escolar. 
http://doi.org/10.15359/ree.25-2.10

http://www.una.ac.cr/educare

educare@una.ac.cr

Ruiz-Juan y Piéron (2013) afirman que el sexo es determinante en la percepción del estudiantado: los hombres más activos que las mujeres en la actividad física escolar y extraescolar (Hughes et al., 2008).

Además, la percepción del estudiantado respecto a la actividad física se ve favorecida por el apoyo que esta recibe de la sociedad. Los estudios de González y Portolés (2014) y Piéron et al. (2008) indican que cuanto más se efectúa la práctica de la actividad física dentro de la jornada escolar o posterior a ella, mayores son las actitudes positivas hacia esta misma. Así tambien, el estudiantado que practica distintas actividades deportivas tendrá una mayor predisposición y valorarán, de manera positiva, las clases de educación física, ejecutándola con mayor facilidad (Hellín y Moreno, 2001a, b; Piéron et al., 2008).

Moreno y Hellín (2001) determinan que el personal docente es otro factor influyente en la enseñanza-aprendizaje del estudiantado. Su sexo o su carácter pueden influenciar la percepción que tiene de la educación física, así como en la satisfacción que este reporta. La influencia del personal docente es determinante para la confección de ambientes en los que se favorece la competencia y autonomía del alumnado (Cox y Williams, 2008). No obstante, en relación con la percepción de la actividad física y la influencia del personal docente por sobre el estudiantado ha sido contrastada por varios autores y autoras (Gaviria-Cortés y Castejón-Oliva, 2019; PortilloTorres et al., 2016; Ramírez-Díaz e Hidalgo-Solano, 2018; Seabra et al., 2011); sin embargo, no se conocen estudios de estas características en el contexto chileno.

Finalmente, se encuentran factores que generan actitudes negativas por parte del alumnado hacia la clase de educación física (Martínez Baena, 2012), destaca el índice de masa corporal (IMC), obesidad-sobrepeso, enfermedad, historial de lesiones, depresión y estrés, entre otros.

Por tanto, el objetivo principal del presente estudio es determinar la influencia del docente, la metodología y el contenido de las clases sobre la percepción del estudiantado chileno acerca de la educación física. Además, como objetivo secundario se pretende determinar la influencia del sexo y el tipo de centro educativo sobre la percepción del estudiantado acerca de la clase de educación física.

\section{Metodología}

\section{Participantes y procedimiento}

La investigación se define a partir de un diseño no experimental, de corte transversal y alcance descriptivo. La muestra se ha constituido por 660 estudiantes de NB5 y NB6 pertenecientes a tres centros educativos: un colegio municipal (financiando a través de aportes fiscales), un subvencionado (subsidio fiscal por estudiante) y un particular (financiado con el cobro de matrícula), pertenecientes a la provincia de Valparaíso, región de Valparaíso, Chile. 
http://doi.org/10.15359/ree.25-2.10

La muestra según el sexo, se distribuyó en $66,06 \%(n=436)$ por mujeres y 33,94\% $(n=224)$ por hombres, con una edad comprendida entre 12 y 13 años, tal como muestra la Tabla 1.

Tabla 1: Muestra de estudiantes pertenecientes a los niveles básicos 5 y 6 de los establecimientos municipal, subvencionado y particular

\begin{tabular}{clcc}
\hline \multirow{2}{*}{ Sexo } & & $\mathrm{n}$ & $\%$ \\
\hline & Hombre & 224 & 33,94 \\
& Mujer & 436 & 66,06 \\
\hline \multirow{2}{*}{ Tipo centro educativo } & Municipal & 210 & 31,81 \\
& Subvencionado & 214 & 32,42 \\
& Particular & 236 & 35,75 \\
\hline
\end{tabular}

Nota: Elaboración propia.

La selección de participantes se ha llevado a cabo mediante muestreo no probabilístico por conveniencia, considerando a escolares de $7^{\circ}$ y $8^{\circ}$ año de Educación General Básica (NB5 y NB6), según establecimientos educativos: municipales $(31,81 \%)$, subvencionados $(32,42 \%)$ y particulares $(35,75 \%)$.

El estudiantado, así como sus tutores o tutoras, han recibido información sobre el objetivo y el tratamiento de datos de la investigación, y han firmado el consentimiento informado de manera voluntaria.

\section{Instrumento}

El cuestionario extraído de Gamboa Saldivar et al. (2009) mide la percepción del estudiantado sobre la clase de educación física y consta de las siguientes 3 dimensiones con cuatro ítems cada una: satisfacción por las actividades deportivas; la clase de educación física y su contenido; y, la función del docente en relación con la clase. Los ítems de la dimensión de satisfacción por las actividades deportivas se consultaron mediante una escala de 4 puntos en la que 1=Totalmente de acuerdo, 2=De acuerdo, 3=En desacuerdo y 4=Totalmente en desacuerdo; mientras que los ítems de las dimensiones de la clase de educación física y su contenido y la función docente en relación con la clase se consultaron utilizando una escala de Likert de 5 puntos ( $1=$ nada; $2=$ poco; $3=$ suficiente; $4=$ bastante y $5=$ mucho) para su respuesta.

De estos 12 ítems es posible lograr tres aspectos esenciales. Los cuatro primeros indagan sobre la satisfacción del estudiantado por las actividades deportivas, pudiendo determinar, a partir de ellos, la percepción sobre el interés generado por las actividades físico-deportivas. Las cuatro siguientes abordan la percepción sobre la clase de educación física y su contenido, las 
http://doi.org/10.15359/ree.25-2.10

http://www.una.ac.cr/educare

educare@una.ac.cr

cuales hacen que el grupo de estudiantes reflexione sobre su experiencia en las clases de esta materia. Y, finalmente los últimos cuatro ítems recogen la percepción que tiene el estudiantado sobre el docente o la docente en relación con su clase.

\section{Análisis de datos}

Los resultados obtenidos del cuestionario se han analizado mediante estadística descriptiva e inferencial, utilizando el software IBM SPSS Statistics versión 25. Se extrajeron estadísticos descriptivos como la media y la desviación típica para el análisis de las variables. También se utilizaron las pruebas de comparación de medias t para muestras independientes, con aplicación previa de prueba de Levene homogeneidad de las varianzas, y ANOVA de un Factor para conocer las diferencias en función del tipo de establecimiento educativo. Para conocer las diferencias significativas entre el estudiantado de cada centro, se utilizó la prueba post hoc de Bonferroni y la deTamhane cuando no existía una distribución homogénea de las varianzas. También se observó el tamaño del efecto mediante la $d$ de Cohen para la prueba t de muestras independientes y el valor de beta al cuadrado en el caso de la prueba ANOVA de un factor.

\section{Resultados}

\section{Percepción general sobre la educación física}

En primer lugar, se analizaron los resultados derivados de la percepción general de las escolares y los escolares chilenos encuestados. En la Tabla 2 podemos observar las medias y desviaciones típicas para cada uno de los indicadores de las tres dimensiones. Tal y como se puede apreciar en la dimensión de satisfacción, el ítem referido a"me gusta la actividad deportiva y la practico siempre que puedo" presenta una puntuación con una tendencia hacia el acuerdo, ya que el valor medio está próximo al valor 3 de la escala $(M=2.8 ; D T=.89)$, que significa que la mayoría de estudiantes están de acuerdo. El ítem que presenta una media más reducida es el referido a "me encanta la actividad deportiva, pero solo para verla como espectador" con una media de1.89 (DT=1.09).

En el caso de la dimensión referida al contenido de las clases de educación física, los ítems que presentan una puntuación más destacada son el número $2(M=3.97 ; D T=1.04), 3(M=4.13$; $\mathrm{DT}=.99)$ y $4(\mathrm{M}=3.96$; $\mathrm{DT}=1.15)$ que indicarían una tendencia positiva en las valoraciones del estudiantado, ya que presentan valores cercanos o superiores al valor 4 de la escala que significa bastante. Por lo tanto, en este caso, la mayoría del estudiantado manifiesta que progresa bastante en sus habilidades tras las clases de educación física, observa la relación entre las actividades y la evaluación final, se siente a gusto con el clima de la clase y se divierte.

Por último, en el caso de la función del docente o la docente en relación con la clase también se observan puntuaciones elevadas en todos los indicadores con valores que superan 
http://doi.org/10.15359/ree.25-2.10

o están cercanos al valor 4 de la escala que significa bastante. Así, comprobamos que la mayoría de las estudiantes indican que su docente les corrige los errores realizados en la práctica ( $M=4.13$; DT=.99), incentiva el espíritu competitivo y el juego limpio ( $M=3.94$; $D T=1.09)$, promueve el trabajo en equipo y el respeto $(M=4.24 ; D T=.93)$ y los ejercicios planteados tienen una dificultad progresiva ( $M=3.59 ; \mathrm{DT}=1.21)$.

Tabla 2: Percepción del estudiantado de los tres centros sobre la educación física

\begin{tabular}{|c|c|c|}
\hline & Media & DT \\
\hline \multicolumn{3}{|l|}{ Satisfacción por las actividades deportivas } \\
\hline 1. Me gusta la actividad deportiva y la practico siempre que puedo. & 2.80 & .89 \\
\hline 2. Solo practico la actividad deportiva por obligación & 2.06 & 1.13 \\
\hline 3. Me encanta la actividad deportiva, pero solo para verla como persona espectadora. & 1.89 & 1.09 \\
\hline $\begin{array}{l}\text { 4. Me gusta la actividad deportiva, pero la practico muy poco porque debo dedicar mi tiempo a } \\
\text { otras actividades. }\end{array}$ & 2.40 & 1.09 \\
\hline
\end{tabular}

La clase de educación física y su contenido

1. ¿Al término de las clases de educación física, notas el progreso en el desarrollo de tus habilidades para realizar los ejercicios? (Recuerda TODAS tus clases de educación física

2. ¿Las actividades en las clases de educación física, tienen relación de continuidad y te ayudan a realizar el ejercicio final que será evaluado a futuro?

3. ¿Te sientes a gusto con el ambiente en el que se desarrolla la clase de educación física? (Relación estudiante-estudiante, y relación docente-estudiante)

4. Según tu punto de vista, ¿en las clases de educación física consideras que te diviertes?

La función del docente en relación con la clase

1. ¿El profesorado de educación física, corrige los errores realizados por el alumnado en la práctica de los ejercicios durante la clase?

2. ¿El profesorado de educación física, incentiva el espíritu competitivo y juego limpio en sus clases? 3.94

3. ¿El profesorado de educación física en sus actividades y ejercicios, promueve el trabajo en equipo y el respeto por el otro/otra?

4. ¿Los ejercicios impuestos por el profesorado de educación física tienen una dificultad progresiva? $3.59 \quad 1.21$

Nota: Elaboración propia. 
http://doi.org/10.15359/ree.25-2.10

http://www.una.ac.cr/educare

educare@una.ac.cr

\section{Influencia del sexo}

A continuación, en la Tabla 3 se muestran los resultados de la influencia del sexo en la percepción sobre la asignatura de educación física. Se puede observar que existen diferencias estadísticamente significativas $(p<.05)$ en el caso del indicador número 2 y 4 de la dimensión de satisfacción por las actividades deportivas. Los hombres presentan una media significativamente más alta $(M=2.22 ; D T=1.19)$ que las chicas $(M=1.98 ; D T=1.10)$ para el ítem relacionado con "solo practico la actividad deportiva por obligación" y significativamente más reducida para el ítem "me gusta la actividad deportiva, pero la practico muy poco porque debo dedicar mi tiempo a otras actividades" con una media de 2.50 (DT=1.10) en el caso de las mujeres y 2.19 (DT=1.05).

En la dimensión relacionada con el contenido de la clase de educación física las puntuaciones medias que informaron diferencias estadísticamente significativas son las referidas a los indicadores 2 y 3. Los dos ítems presentan medias significativamente más altas en los hombres que en las mujeres, con valores de 4.17 (DT=.88) para los chicos y 3.87 (DT=1.1.0) para las mujeres en el indicador referido a la relación entre las actividades de las clases y la evaluación; mientras que muestra una media de 4.31 (DT=.87) para las chicos y 4.03 (DT=1.03) para las chicas en el caso del indicador relacionado con el ambiente en la clase de educación física.

Por último, en la dimensión referida a la función del personal docente, las puntuaciones medias que mostraron diferencias estadísticamente significativas son las referidas a los indicadores 2 y 4 . En el caso del ítem 2 la media de las mujeres fue significativamente más alta $(M=4.05)$ que la de los hombres $(M=3.73)$, mientras que en el ítem 4 se observó la tendencia inversa $(M=3.49$ y 3.80 , respectivamente).

Tabla 3: Percepción del estudiantado sobre la educación física según el sexo

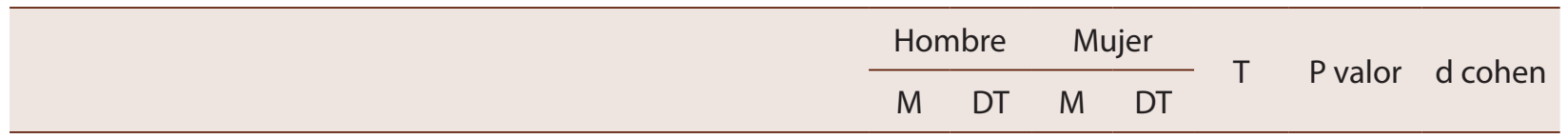

Satisfacción por las actividades deportivas

1. Me gusta la actividad deportiva y la practico siempre que puedo.

$\begin{array}{llllll}2.81 & .86 & 2.80 & .90 & .19 & .85\end{array}$

$\begin{array}{llllll}2.22 & 1.19 & 1.98 & 1.10 & 2.46 & .01^{*}\end{array}$

.21

3. Me encanta la actividad deportiva, pero solo para verla como persona espectadora.

$\begin{array}{llllll}1.82 & .93 & 1.92 & 1.15 & -1.25 & .21\end{array}$

$\begin{array}{llllll}2.19 & 1.05 & 2.50 & 1.10 & -3.57 & <.001^{* * *}\end{array}$ 
http://doi.org/10.15359/ree.25-2.10

La clase de educación física y su contenido

1. ¿Al término de las clases de educación física, notas elprogreso en el desarrollo de tus habilidades para realizar los ejercicios? $\begin{array}{lllllll}3.45 & 1.09 & 3.53 & 1.14 & -.87 & .39\end{array}$ (Recuerda TODAS tus clases de educación física)

2. ¿Las actividades en las clases de educación física tienen relación de continuidad y te ayudan a realizar el ejercicio final que será evaluado a futuro?

$\begin{array}{lllllll}4.17 & .88 & 3.87 & 1.10 & 3.72 & <.001^{* * *} & .29\end{array}$

3. ¿Te sientes a gusto con el ambiente en el que se desarrolla la clase de educación física? (Relación estudianteestudiante, y relación docente- estudiante)

$\begin{array}{lllllll}4.31 & .87 & 4.03 & 1.03 & 3.67 & <.001^{* * *} & .29\end{array}$

4. Según tu punto de vista, ¿en las clases de educación física consideras que te diviertes?

La función del docente en relación con la clase

1. ¿El profesorado de educación física, corrige los errores realizados por el alumnado en la práctica de los ejercicios durante la clase?

$\begin{array}{llllll}4.05 & .91 & 4.17 & 1.03 & -1.57 & .12\end{array}$

2. ¿El profesorado de educación física, incentiva el espíritu competitivo y juego limpio en sus clases?

$\begin{array}{lllllll}3.73 & 1.20 & 4.05 & 1.02 & -3.37 & .001^{* * *} & -.30\end{array}$

3. ¿El profesorado de educación física en sus actividades y $\begin{array}{lllllll}\text { ejercicios, promueve el trabajo en equipo y el respeto por } & 4.20 & .91 & 4.26 & .94 & -.80 & .42\end{array}$ el otro?

4. ¿Los ejercicios impuestos por el profesorado de educación física tienen una dificultad progresiva?

Nota: $M=$ Media; DT=Desviación típica; ${ }^{*}$ indica diferencias estadísticamente significativas a nivel $p<.05 ;{ }^{* *} p<.01$; ${ }^{* * *} \mathrm{p}<.001$. Elaboración propia.

\section{Influencia del tipo de centro}

En la Tabla 4 se muestran los resultados de la prueba ANOVA de un factor para observar las diferencias significativas entre las puntuaciones medias de los indicadores. Como podemos observar todos los indicadores presentaron diferencias estadísticamente significativas $(p<.01)$ en función del centro en el que estaban escolarizados. 
http://doi.org/10.15359/ree.25-2.10

http://www.una.ac.cr/educare

educare@una.ac.cr

Según las pruebas post hoc de comparaciones múltiples, en la dimensión de satisfacción con la práctica deportiva, se observaron diferencias estadísticamente significativas entre los escolares y las escolares de los tres centros en los indicadores 2 y 3 , mientras que en el ítem 1 las diferencias se observaron al comparar el centro municipal con los otros dos centros y en el ítem 4 al comparar el centro subvencionado con los otros dos.

Las puntuaciones medias mostraron valores más elevados para los escolares y las escolares del centro municipal en el caso del indicador 1 y 4, mientras que los valores medios más reducidos se observaron los indicadores 2 y 3 . En el centro subvencionado destacan los ítems 2 y 3 con una media más alta que en el resto de los centros. Los resultados muestran que el estudiantado del centro municipal es el que muestra una mayor tendencia hacia el acuerdo respecto al interés por la actividad deportiva y su práctica $(M=3.15 ; D T=.86)$, observándose la tendencia inversa en los aspectos relacionados con la obligación por la práctica deportiva $(M=1.42 ; D T=.73)$ y el interés por verla como espectador $(M=1.50 ; D T=.90)$. Los escolares del centro subvencionado muestran una tendencia hacia el acuerdo $(M=2.70 ; D T=1.16)$ en el ítem referido a la obligación como motivo para practicar deporte.

En la dimensión de contenido de educación física, las pruebas post hoc mostraron que las diferencias significativas se observaron entre el estudiantado del centro municipal y el particular en los ítems 1 y 4, mientras que entre los escolares y las escolares del centro municipal y el subvencionado para el ítem 2 y 3 también se observaron diferencias significativas al comparar el centro subvencionado con el particular en los ítems 1 y 4. Los resultados de las puntuaciones muestran que el centro municipal presenta la media más alta para el indicador relacionado con el progreso en el desarrollo de habilidades en los escolares y las escolares al finalizar la clase de educación física $(M=3.68$; $\mathrm{DT}=1.06)$, mientras que los escolares y las escolares del centro particular muestran una media más reducida en este indicador $(M=3.24 ; D T=1.08)$. Por otro lado, los escolares y las escolares del centro subvencionado presentan medias más altas para los aspectos relacionados con la relación entre las actividades y la evaluación final ( $M=4.13 ; D T=.96)$, el ambiente de aprendizaje ( $M=4.32 ; \mathrm{DT}=.94)$ y la diversión en las clases $(M=4.16 ; \mathrm{DT}=1.05)$.

Por último, en la dimensión de la función del personal docente en relación con la clase se comprobó que solo se observaron diferencias significativas entre escolares de los tres centros para el ítem 3, mientras que en los ítems 1, 2 y 4 se comprobó que existían diferencias significativas entre el estudiantado del centro municipal y el particular y entre el subvencionado y el particular. Los resultados muestran que el estudiantado del centro subvencionado presenta puntuaciones medias más altas en los indicadores referidos a la corrección de los ejercicios por parte del profesorado ( $M=4.31$; DT=.85), incentiva la competición y el juego limpio $(M=4.25$; $\mathrm{DT}=.95)$ y promueve el trabajo en equipo y el respeto por el otro sujeto $(M=4.49 ; \mathrm{DT}=.85)$. 
http://doi.org/10.15359/ree.25-2.10

http://www.una.ac.cr/educare educare@una.ac.cr

Tabla 4: Percepción del estudiantado sobre la educación física según el tipo de centro en el que están escolarizados

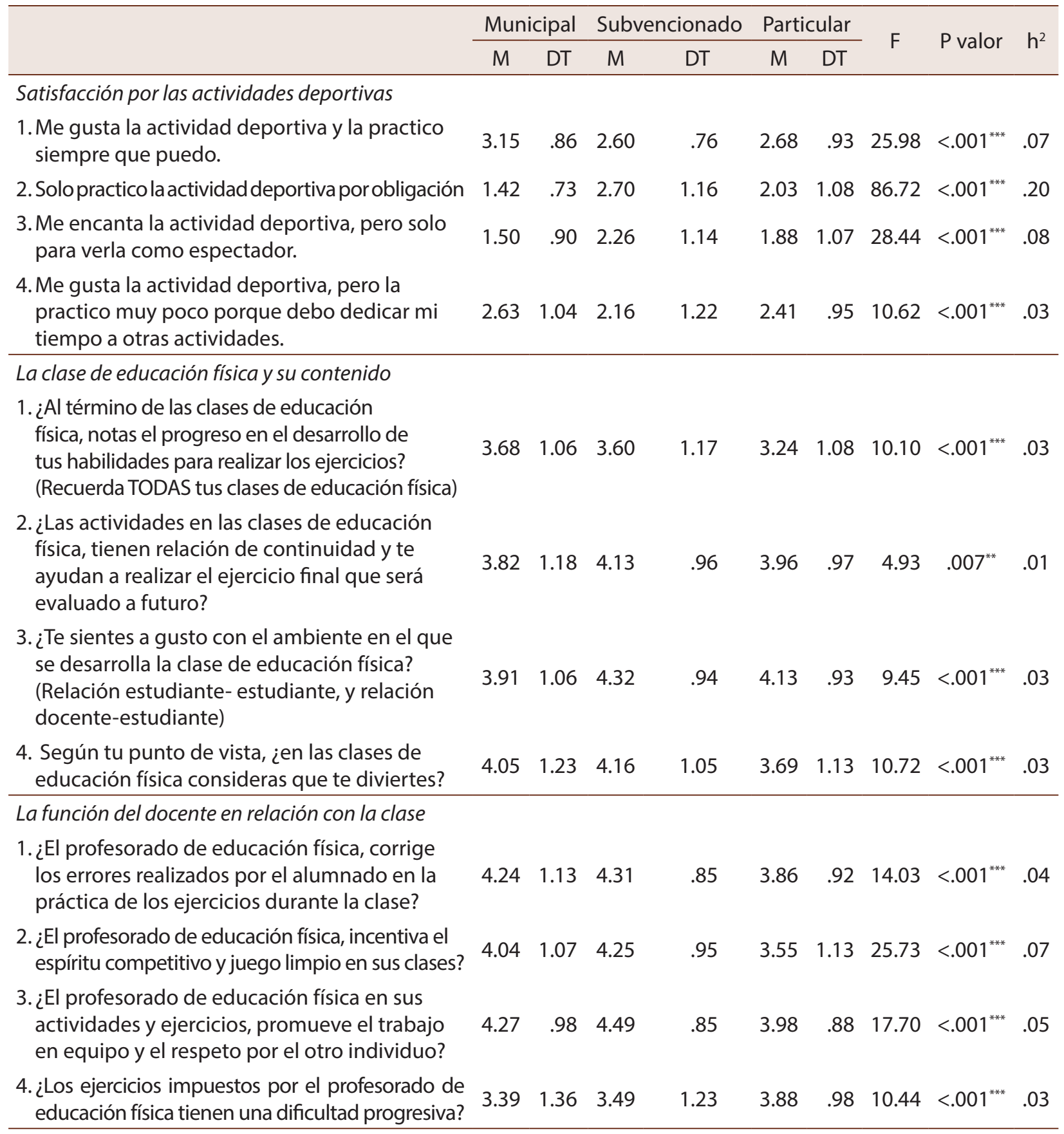

Nota: $\mathrm{M}=$ Media; DT=Desviación típica; ${ }^{* *}$ indica diferencias estadísticamente significativas a nivel $\mathrm{p}<.01 ;{ }^{* * *} \mathrm{p}<.001$. Elaboración propia. 
http://doi.org/10.15359/ree.25-2.10

http://www.una.ac.cr/educare

educare@una.ac.cr

\section{Discusión}

Sallis et al. (2012) destacan la influencia de la educación física en la percepción del estudiantado sobre la actividad física (escolar y extraescolar), pues tiene repercusión en la generación de estilos de vida activos y saludables por parte del estudiantado. Sobre esto, la muestra reporta valores medio-altos $(M=3,96)$ en cuanto a la percepción de la diversión cuando llevan a cabo la práctica, tal sentimiento podría influir positivamente en la creación de hábitos de vida saludables. En relación con la práctica deportiva, las personas participantes encuestadas reportan satisfacción media con la práctica $(M=2,8)$, según el estudiantado perteneciente al centro municipal, las actividades deportivas generan más gusto y la practican siempre que pueden en un mayor nivel $(M=3,15)$ respecto de los centros subvencionado y particular $(M=2,6$ y $M=2,68$, respectivamente). En este sentido, es importante la generación en el estudiantado de actitudes positivas hacia la práctica de actividad física, de vital importancia para un correcto desarrollo motriz (Piéron et al., 2008). En esta misma línea, la generación de actitudes positivas hacia la práctica es crucial en la educación física, ya que gran cantidad de estudios (González y Portolés, 2014; Piéron et al., 2008) confirma que el incremento en la frecuencia de práctica semanal produce mejoras en la percepción que se tiene hacia esta, independientemente de si esta se lleva a cabo en el centro o fuera de él. Además, la práctica con regularidad permite al estudiantado llevar a cabo las actividades con mayor facilidad (Hellín y Moreno, 2001a, b; Piéron et al, 2008), lo que permitiría, además de un incremento en las sesiones semanales de actividad, una mayor adherencia, lo cual resulta determinante para la salud del estudiantado.

La adherencia a la práctica deportiva está muy influenciada por la satisfacción que reporta el estudiantado al finalizar las sesiones y unidades didácticas. Teniendo en cuenta que el estudiantado de los centros municipal y subvencionado $(M=3,68$ y $M=3,6)$, manifiestan una positiva tendencia respecto a la percepción del progreso en el desarrollo de habilidades para realizar ejercicios, al mejorar respecto a los objetivos establecidos al inicio de la programación docente. En el caso del estudiantado del centro particular se reporta una percepción sobre el progreso a término de la clase inferior $(M=3,24)$ que en los otros centros.

Otros aspectos relacionados con la clase de educación física y su contenido, como la relación entre las actividades y con la evaluación futura, los resultados informaron tendencias positivas en la muestra. La percepción del grupo de alumnos hombres $(M=4,17)$ es mayor que en el de mujeres $(M=3,87)$. Por lo tanto, estos resultados nos permiten relevar la importancia que tiene la implicación del estudiantado en la clase es determinante en la consecución de los objetivos, cuestión que es reafirmada por Moreno-Murcia et al. (2011), quienes destacan la importancia de que el estudiantado mantenga altos niveles de motivación intrínseca a lo largo de todo el curso. Tal necesidad deriva del impacto que dicha motivación tiene sobre la percepción y disfrute del estudiantado, pues puede causar efectos adversos cuando esta no es intrínseca (Sánchez-Oliva et al., 2015). Este factor se ve influenciado por uno de los agentes más determinantes dentro del proceso de enseñanza-aprendizaje: el personal docente. 
http://doi.org/10.15359/ree.25-2.10

La relevancia del profesorado está determinada por la capacidad que este tiene para influir sobre la percepción del estudiantado acerca de la práctica deportiva (Moreno y Hellín, 2001). Sobre esto, el estudiantado de los tres centros educativos señala sentirse a gusto con el ambiente en que se desarrolla la clase de educación física $(M=4,13)$, mientras que respecto a la variable sexo, existe una diferencia manifiesta en torno al ambiente percibido, donde los hombres $(M=4,31)$ informan una valoración mayor que el grupo de mujeres $(M=4,03)$, aunque ambas medias son altas, lo cual se puede atribuir a que el profesorado de educación física es considerado como la fuente principal de motivación para sus estudiantes, ya que es quien propicia situaciones que actualizan las necesidades del grupo. De este modo, el profesorado puede aplicar estrategias motivacionales antes, durante y después de la clase y repercutir, con ello, en el aprendizaje y el desempeño de sus estudiantes. Por otro lado, estudiantes de los diferentes establecimientos (municipal, subvencionado y particular) reportan una positiva influencia respecto a la promoción, por parte del profesorado, respecto al trabajo en equipo y respecto por los compañeros y compañeras ( $M=4,17 ; M=4,49$ y $M=3,98$ respectivamente), valores significativos debido a la importancia que presentan las relaciones entre estudiantes para la consecución de un clima de clase óptimo y armónico en el que se produzca la mejora de las capacidades motrices y socio-afectivas (Holt et al., 2012). En definitiva, la motivación estudiantil depende, en gran medida, de cómo el alumnado perciba el clima motivacional que su docente presente en las clases (Duda, 2001; Papaioannou et al., 2004).

\section{Conclusiones}

El objetivo del presente trabajo fue conocer la influencia del personal docente, la metodología y el contenido de las clases de educación física en la percepción del estudiantado sobre esta misma, y establecer las posibles diferencias, en función del sexo del estudiantado y el tipo de establecimiento educativo (municipal, subvencionado y particular), en caso de que existiesen.

Como primera conclusión se ha podido establecer que el grupo de hombres manifiesta una mayor satisfacción al momento de ejecutar una actividad deportiva, aunque estas, en ocasiones, deben ejecutarlas por obligación o porque deben dedicarle tiempo a otro tipo de actividades, no obstante, se preocupan de las actividades que están realizando y cómo serán evaluadas. Otro punto relevante para destacar de este grupo, es que considera importante el ambiente de las clases de educación física y las relaciones interpersonales estudiante-estudiante, docente-estudiante. Sin embargo, para el grupo de mujeres es mucho más significativo que el profesorado de educación física incentive el espíritu competitivo y el juego limpio en sus clases.

Por otro lado, los resultados han evidenciado diferencias estadísticamente significativas del establecimiento particular respecto a los centros subvencionado y municipal, resulta menor la satisfacción reportada por el estudiantado del primer centro educativo en cuanto a 
http://doi.org/10.15359/ree.25-2.10

http://www.una.ac.cr/educare

educare@una.ac.cr

la clase de educación física. Estos valores podrían estar influenciados por la continuidad de la sesión, la retroalimentación aportada por el personal docente, así como la actitud de este mismo ante las clases de educación física, ya que los resultados obtenidos en estas variables son significativamente menores en el establecimiento particular.

Además de lo anterior, el personal docente es fundamental en el proceso de enseñanzaaprendizaje, ya que en los centros en los que la retroalimentación se presenta de manera positiva y se muestra continuidad en las sesiones y las clases son orientadas a la consecución de los objetivos planteados, el estudiantado mostró niveles significativamente superiores de satisfacción y adherencia a la práctica deportiva.

Por último, la metodología implementada por el personal docente parece ser el factor de menor relevancia en la percepción del estudiantado acerca de la clase de educación física, esto puede deberse a que todo el personal docente de la investigación utiliza métodos basados en la participación activa del estudiantado, así como la búsqueda de soluciones, y desecha los métodos tradicionales que pueden crear un mayor rechazo a la práctica deportiva.

Considerando los resultados obtenidos en la presente investigación y con el objetivo de poder generalizarlos, sería importante considerar en futuras investigaciones: incrementar del tamaño de la muestra que involucre estudiantes pertenecientes a otros niveles académicos; tomar en cuenta otras variables que pueden influir en la percepción del estudiantado sobre la clase de educación física y, finalmente, comparar los resultados obtenidos con diferentes lugares geográficos, y comparar y establecer semejanzas y diferencias en las estrategias educativas implementadas.

\section{Implicaciones prácticas}

Los resultados de este estudio permiten al personal docente y directivo de los centros educativos de Chile en los que se imparte educación física incrementar la información sobre la relación existente entre estudiante-estudiante, docente-estudiante, en función de los procesos de enseñanza-aprendizaje que son llevados a cabo durante la clase. Esto podría ayudar a mejorar las actuaciones aplicadas por los diferentes agentes participantes en el proceso educativo (docentes, estudiantes, personal directivo, entre otros) destinados a la mejora de la percepción del estudiantado, con el fin de generar y reforzar hábitos de vida saludables, así como adherencia a la práctica deportiva, principales objetivos de la educación física.

\section{Referencias}

Cox, A. y Williams, L. (2008). The roles of perceived teacher support, motivational climate, and psychological need satisfaction in students' physical education motivation. Journal of Sport and Exercise Psychology, 30(2), 222-239. https://doi.org/10.1123/jsep.30.2.222 
http://doi.org/10.15359/ree.25-2.10 http://www.una.ac.cr/educare educare@una.ac.cr

Duda, J. L. (2001). Achievement goal research in sport: Pushing the boundaries and clarifying some misunderstandings. En G. C. Roberts y D. C. Treasure (Eds.), Advances in motivation in sport and exercise (pp. 129-182). Human Kinetics. https://www.scirp.org/ (S(oyulxb452alnt1aej1 nfow45))/reference/ReferencesPapers.aspx?ReferencelD=2035110

Gamboa Saldívar, J, Ibarra Martínez, J., Leiva Soto, G. y Osorio Lorca, T. (2009). Opinión de los alumnos y alumnas de colegios particulares no subvencionados en los niveles NB5 y NB6, de las clases de educación física [Tesis doctoral]. Universidad Andrés Bello, Chile. http:// repositorio.unab.cl/xmlui/handle/ria/8476

Gaviria-Cortés, D. f. y Castejón-Oliva, F. J. (2019). ¿Qué aprende el estudiantado de secundaria en la asignatura de educación física? Revista Electrónica Educare, 23(3), 1-20. https://doi. org/10.15359/ree.23-3.2

González Correa, A. M. y González Correa, C. H. (2010). Educación física desde la corporeidad y la motricidad. Hacia la Promoción de la Salud, 15(2), 173-187. http://www.scielo.org.co/pdf/ hpsal/v15n2/v15n2a11.pdf

González, J. y Portolés, A. (2014). Actividad física extraescolar: Relaciones con la motivación educativa, rendimiento académico y conductas asociadas a la salud. Revista Iberoamericana de Psicología del Ejercicio y el Deporte, 9(1), 51-65. https://www.redalyc.org/ pdf/3111/311130199005.pdf

González Suárez, Á. M. y Otero Parra, M. (2005). Actitudes de los padres ante la promoción de la actividad física y deportiva de las chicas en edad escolar. Cuadernos de psicología del deporte, 5(1-2), 173-195. https://revistas.um.es/cpd/article/view/93481

Hellín, P. y Moreno, J. A. (2001a). Actitudes hacia la educación física de los alumnos de ciclos formativos. Actas del II Congreso de ciencias de la actividad física y el deporte (pp. 549-557). Valencia: Universidad de Valencia.

Hellín, P. y Moreno, J. A. (2001b). Importancia de la educación física. Actas del II Congreso internacional de educación física y diversidad (pp. 693-708). Murcia: Consejería de Educación y Universidades.

Holt, N. L., Sehn, Z., Spence, J. C., Newton, A. S. y Ball, G. D. C. (2012). Physical education and sport programs at an inner city school: Exploring possibilities for positive youth development. Physical education and sport pedagogy, 17(1), 97-113. https://doi.org/10.1080/17408989.2 010.548062

Hughes, J. P., McDowell, M. A. y Brody, D. J. (2008). Leisure-Time physical activity among US adults 60 or more years of age: Results from NHANES 1999-2004. Journal of Physical Activity and Health, 5(3), 347-358. https://doi.org/10.1123/jpah.5.3.347 
http://doi.org/10.15359/ree.25-2.10

http://www.una.ac.cr/educare

educare@una.ac.cr

Kane, J. E. (2015) (Ed.). Psychological aspects of physical education and sport. Routledge. https:// doi.org/10.4324/9781315625140

Martínez Baena, A. C. (2012). Factores influyentes en la actividad físico-deportiva de adolescentes españoles: Opiniones sobre el papel de la escuela [Tesis doctoral]. Universidad de Granada, España. https://digibug.ugr.es/handle/10481/21764

Méndez-Giménez, A., Fernández-Río, J., Cecchini Estrada, J. A. y González González de Mesa, C. (2013). Perfiles motivacionales y sus consecuencias en educación física. Un estudio complementario de metas de logro $2 \times 2$ y autodeterminación. Revista de psicología del deporte, 22(1), 29-38. https://www.redalyc.org/pdf/2351/235127552005.pdf

Mendiara Rivas, J. (2008). La psicomotricidad educativa: Un enfoque natural. Revista Interuniversitaria de Formación del Profesorado, 22(2), 199-220. https://www.redalyc.org/ pdf/274/27414780012.pdf

Ministerio de Educación. (12 de setiembre, 2009). Ley Núm 20.370 establece la Ley General de Educación. Diario Oficial de la República de Chile. https://www.bcn.cl/leychile/ navegar?idNorma $=1006043$

Moreno, J. A. y Hellín, P. (2001). Valoración de la educación física por el alumno según el género del profesor. En Actas del XIX Congreso nacional de educación física y escuelas universitarias de magisterio (pp. 1267-1275). Universidad de Murcia.

Moreno-Murcia, J. A., Sicilia, A., Cervelló, E., Huéscar, E. y Dumitru, D. C. (2011). The relationship between goal orientations, motivational climate and selfreported discipline in physical education. Journal of Sports Science and Medicine, 10(1), 119-129. https://www.ncbi.nlm. nih.gov/pmc/articles/PMC3737903/

Papaioannou, A., Marsh, H. W. y Theodorakis, Y. (2004). A multilevel approach to motivational climate in physical education and sport settings: An individual or a group level construct? Journal of Sport and Exercise Psychology, 26(1), 90-118. https://doi.org/10.1123/jsep.26.1.90

Parlebas, P (2001). Juegos, deportes y sociedades: Léxico de praxiología motriz. Paidotribo.

Piéron, M., Ruiz Juan, F. y García Montes, M. E. (2008). La opinión del alumnado de enseñanza secundaria sobre las clases de educación física: Un desafío para los profesores y los formadores. Revista Fuentes, 8, 159-175 https://idus.us.es/handle/11441/32340

Portillo-Torres, M., Hernández-Quesada, Ó. y Quirós-Quirós, H. (2016). Opinión de docentes y estudiantes sobre las clases de educación física en secundaria. Revista Electrónica Educare, 20(2), 1-23. https://doi.org/10.15359/ree.20-2.5 
http://doi.org/10.15359/ree.25-2.10

Ramírez-Díaz, J. L. e Hidalgo-Solano, F. (2018). Satisfacción de estudiantes de secundaria nocturna y su incidencia en el abandono escolar. Revista Electrónica Educare, 22(1), 1-14. https://doi.org/10.15359/ree.22-1.14

Rosa Guillamón, A., García Cantó, E. y Carrillo López, P. J. (2018). La educación física como programa de desarrollo físico y motor. Revista digital de Educación Física, 9(52), 105-124. https://dialnet.unirioja.es/servlet/articulo?codigo $=6408944$

Ruiz-Juan, F. y Piéron, M. (2013). Orientaciones de meta en educación física y nivel de actividad físico-deportiva en estudiantes mexicanos. Universitas psychologica, 12(1), 235-247. https://www.redalyc.org/pdf/647/64728729021.pdf

Sallis, J. F., McKenzie, T. L., Beets, M. W., Beighle, A., Erwin, H. y Lee, S. (2012). Physical education's role in public health. Steps forward and backward over 20 years and hope for the future. Research Quarterly for Exercise and Sport, 83(2), 125-135. https://doi.org/10.1080/0270136 $\underline{7.2012 .10599842}$

Sánchez-Oliva, D., Leo Marcos, F. M., Alonso, D. A., Pulido-González, J. J. y García-Calvo, T. (2015). Análisis de los perfiles motivacionales y su relación con los comportamientos adaptativos en las clases de educación física. Revista Latinoamericana de Psicología, 47(3), 156-166. https://doi.org/10.1016/j.rlp.2015.06.007

Sassano, M. y Bottini, P. (2000). Apuntes para una historia de la psicomotricidad. Breve recorrido por los principales referentes, históricos y actuales, de la práctica y los conceptos de la psicomotricidad. P. Bottini (Comp.), Psicomotricidad: Prácticas y conceptos (pp. 13-36). Miño y Dávila editores.

Seabra, A. F., Mendonça, D. M., Thomis, M. A., Malina, R. M. y Maia, J. A. (2011). Correlates of physical activity in portuguese adolescents from 10 to 18 years. Scandinavian Journal of Medicine and Science in Sports, 21(2), 318-323. https://doi.org/10.1111/j.1600-0838.2009.01030.x 